\title{
COST-BENEFIT ANALYSIS OF RAIL TUNNEL INSPECTION FOR PHOTOGRAMMETRY AND LASER SCANNING
}

\author{
F. Panella $\stackrel{1,2, *}{,}$ N. Roecklinger ${ }^{2}$, L. Vojnovic ${ }^{3}$, Y. $_{\operatorname{Loo}^{2}}$, J. Boehm $^{1}$ \\ ${ }^{1}$ UCL, Dept. of Civil, Environmental and Geomatic Engineering, University College London, Gower Street, London, WC1E 6BT UK \\ (fabio.panella.16, j.boehm)@ucl.ac.uk \\ ${ }^{2}$ Ove Arup \& Partners Ltd., 13 Fitzroy Street, London W1T 4BQ \\ (Yung.Loo, Noemi.Roecklinger)@arup.com \\ ${ }^{3}$ Network Rail High Speed Ltd (NRHS), Singlewell Infrastructure Maintenance Depot, Henhurst Rd, Gravesend DA12 3AN \\ Luka.Vojnovic@networkrail.co.uk
}

Commission II, WG II/10

KEY WORDS: Photogrammetry, 3D reconstruction, Laser scanner, Tunnel inspection, Tunnel maintenance, Low-end sensors

\begin{abstract}
:
The civil engineering and construction sector, including the railway industry, is seeking innovative approaches to reduce costs on repetitive and labour-intensive tasks and avoid the use of highly qualified staff for simple manual duties. Such tasks can include the visual inspection of tunnels, where the process is still dominated by manual operations. Our work compares Close Range Photogrammetry (CRP) and Terrestrial Laser Scanning (TLS), both performed with low-end sensors to reflect the industry's tendency towards easy to use and easy to maintain hardware. It also analyses the benefits of substituting conventional visual inspections of tunnels with automated survey approaches and computer vision techniques. The project's outcomes suggest that photogrammetry is a valid alternative to laser scanning for visual inspection of concrete segmentally lined tunnels: from the geometric point of view it provides global accuracy at comparable level to laser scanning, in addition it halves the time to generate the 3D model and provides the user with photo-realistic outputs. It is generally more versatile and it is easier to inspect, visualise and navigate the data. The authors argue that the results presented here will push tunnel inspection in the direction of automated approaches with direct benefits on surveying costs as well as Health \& Safety (H\&S). Utilising available technology supports risk-based asset management and thus ensures safe and operational performance of a railway for passengers to use.
\end{abstract}

\section{INTRODUCTION}

While civil structures are usually designed to last for a specified life-span (Brian, 1997, Gulvanessian, 2009), most tunnels are designed for an indefinite design working life (McKibbins et al., 2009). For that purpose, a strict schedule of inspections is demanded with intervals between consecutive visits depending on the required Level of Details (LoD) (McKibbins et al., 2009).

To date, visual inspections is still dominated by manual operations implicating inspectors working at night in potentially elevated health and safety risk conditions. Hence the aims of innovative approaches for this task are:

- to reduce costs of repetitive and labour-intensive tasks and avoid the use of highly qualified staff for simple manual duties. This will allow to perform inspections with high LoD more frequently, providing the general public with the trust in safe and reliable infrastructure and causing less disruptions;

- to reduce the time spent on site to collect data. This would improve H\&S conditions considering the numerous hazards operators are exposed to in tunnels.

This report presents the findings and outcomes from Arup's project in collaboration with Network Rail High Speed Ltd

\footnotetext{
* Corresponding author
}

(NRHS) which aimed at understanding the benefits of supplementing conventional manual inspection of the HS1 tunnel infrastructure with automated survey approaches and computer vision techniques. Towards this aim, Arup and NRHS have undertaken image-based tunnel inspection (Close-Range Photogrammetry - CRP) and on-site survey work (Terrestrial Laser Scanner - TLS). The inspected infrastructure is a $50 \mathrm{~m}$ section of a $7.15 \mathrm{~m}$ diameter concrete segmentally lined tunnel used for high speed rail traffic. The aim of the project is to assess the benefits using photogrammetry versus the more established (in terms of tunnel inspection) laser scanning technique. The two approaches are compared with each other and with the traditional visual inspection. The comparison takes into consideration several factors including geometric accuracy, repeatability, objectivity, data completeness, accessibility and interpretability. We also consider the end-to-end time per each approach including data acquisition and post processing.

The sensors for both CRP and TLS are selected to reflect construction industry's tendency towards easy to use and easy to maintain hardware. For the CRP approach, the selection of the sensors comes from a multitude of considerations, one of which is the low price that enables an immediate return of the investment and a more agile replacement when damaged, an event to consider when working in such environment. Moreover, the proposed system is versatile and can be adapted to a multitude of cases and it is easy to incrementally upgrade as the sensors develop further. Finally the authors also take into consideration the instrumentation's weight (including the aluminium frame) to guarantee sufficient battery life, to perform the inspection 
and exit the tunnel safely, as well as the stability of the vehicle itself. All these considerations favoured the selection of GoPro as the imaging sensor.

The Leica BLK360 is the sensor selected for the laser scanning data acquisition. It is chosen as, among other requirements, it has an integrated thermal camera, which is considered beneficial for the detection of water ingress in tunnels (Fahmy et al., 2009, Yu et al., 2018). In addition, its relatively low-cost enables a fair comparison with CRP.

The following paragraphs detail the end-to-end pipeline, describes the comparison parameters and summarize the outputs.

\section{RELATED WORKS}

Photogrammetry is a precise measuring and visualization tool used in several areas of application, including civil engineering. Depending on the image capturing technique, it can be classified into aerial, close-range or stereo photogrammetry (Luhmann et al., 2013). The accuracy of the photogrammetric outputs is influenced by several aspects, the most important being image quality (combination of image resolution, pixel size and lighting), camera calibration and camera network. From the several known techniques to determine the interior parameters of the imaging sensor, the present paper employs self calibration. Unlike test field calibration, it determines the camera's interior parameters from the same images used for the actual measurement of the object (Luhmann et al., 2013). Therefore, image observations are used both for maximizing calibration precision and for object point determination. To minimize the calibration error, camera network design is crucial as well (Shortis, 2019): convergent angles between the images is fundamental for the determination of principal distances (Centre, 2003), while a sufficient side and forward overlap increases the redundancy of observations per point improving the precision of the estimation of unknown parameters.

The inspection of tunnels with photogrammetry became increasingly popular in the last decade. In the literature there have been several suggestions for the application of photogrammetry in tunnels and different approaches were suggested to maximize the accuracy of the three dimensional model. (Charbonnier et al., 2013) performs the 3D reconstruction of a canal-tunnel with both laser scanning and photogrammetric techniques collecting several datasets. Considering the terrestrial laser scanner as a reference, they assess the accuracy of the photogrammetric results which spans between 4.1 and $5.6 \mathrm{~cm}$. (Bauer et al., 2015) uses a single-camera based imaging device equipped with a motorized tilt mechanism to acquire a $360^{\circ}$ (vertical) panorama together with a lighting system positioned 1 tunnel diameter behind the camera. A deviation between the laser scanner data and the photogrammetric reconstruction less than $10 \mathrm{~mm}$ is achieved. (Moisan et al., 2017) fully exploit the capacities of photogrammetry to overcome the absence of GPS signal to georeference the data. The difference (in terms of distance) between the photogrammetric and the laser scanned $3 \mathrm{D}$ models ranges between 10 and $37 \mathrm{~mm}$. A combination of laser scanner and photogrammetry is performed in (Paar, Kontrus, 2007). The exported cylindrical orthophoto is then used for manual crack monitoring.

Action cameras are small, lightweight, robust, low cost, and high definition both in video and still images mode. Used primarily for capturing sporting activities, in the last years their low price point has also attracted the construction industry's attention with the aim of high quality image inspections (McDonnell, Devriendt, 2017) or for 3D reconstruction (Balletti et al., 2014a, Reznicek, Luhmann, 2019, Maldonado et al., 2016). In civil engineering and in architecture, the use of fisheye lenses is becoming more and more common (Mandelli et al., 2017). The angle of view of these lenses, commonly over $120^{\circ}$, offers the versatility to capture wide areas with a manageable number of images (Fassi et al., 2018). In (Perfetti et al., 2018) the ability to produce high accuracy 3D model in narrow spaces is documented. These aspects translate in a sensible reduction of the costs. The downside of very wide angle lenses is the difficulty in directly applying the well known Brown's distortion model (Brown, 1971). Several authors deployed their own camera model for calibration purposes. A four-step camera calibration for fisheye lenses is proposed by (Kannala, Br, 2004) while (Perfetti et al., 2018) opt for the approach with self calibration. However, the Brown model is successfully applied (Teo, 2015) to fisheye lenses projections (Agisoft Metashape User Manual - Professional Edition, Version 1.5, 2019) but at the price of a reduction in accuracy of the distortion parameters estimation (Balletti et al., 2014b). Moreover, assessing the applicability of action cameras for photogrammetric purposes, (Wierzbicki, 2018) notices the more consistent repeatability of calibration results performed with Agisoft compared to GML Camera Calibration Toolbox.

LiDAR (Light Detection And Ranging) is the technique to measure distances using light pulses (measurement is made based on the time of flight of the beams) or amplitude modulation (measurement based on the calculation of the phase shift of the returning signal). Such a technique produces high precision 3D measurements (the maximum range of measurement depends on the technology adopted). Application of laser scanning technique is not new in fields like heritage buildings recording (Fort-González et al., 2002, Herrera et al., 2008) and construction inspection (González-Aguilera et al., 2008, Qiu, Gao, 2010, M et al., n.d., Monserrat, Crosetto, 2008). (Lemy et al., 2006) can be considered one of the first groups applying TLS for wall displacement in tunnel monitoring. Applications of TLS for inspection of tunnels is still a topic of active investigation. Detection of water leakage in tunnels is performed in (Yu et al., 2018). They integrate laser point cloud and infrared thermal imaging in order to avoid misclassification due to the presence of multiple material and services applied to the tunnel lining. Instead, (Barla et al., 2016) combines TLS and thermal imagery to map discontinuities in tunnels showing the versatility of such an instrument.

\section{EXPERIMENTAL PROGRAM}

\subsection{Test setup}

This section reports the outcomes for the survey of a $50 \mathrm{~m} \mathrm{sec}-$ tion from a $7.15 \mathrm{~m}$ diameter concrete segmentally lined tunnel for high speed rail traffic. The survey is performed with both Terrestrial Laser Scanning (TLS) and Close-Range Photogrammetry (CRP). TLS technique is used alongside photogrammetry as it is generally recognised as an established and robust method to directly provide reliable and precise $3 \mathrm{D}$ information. The resulting 3D model is regarded as a geometrically highly accurate reference data set for the photogrammetric 3D model. With the aim of comparing the two datasets, a common reference system is established by applying 20 targets to the tunnel lining used 


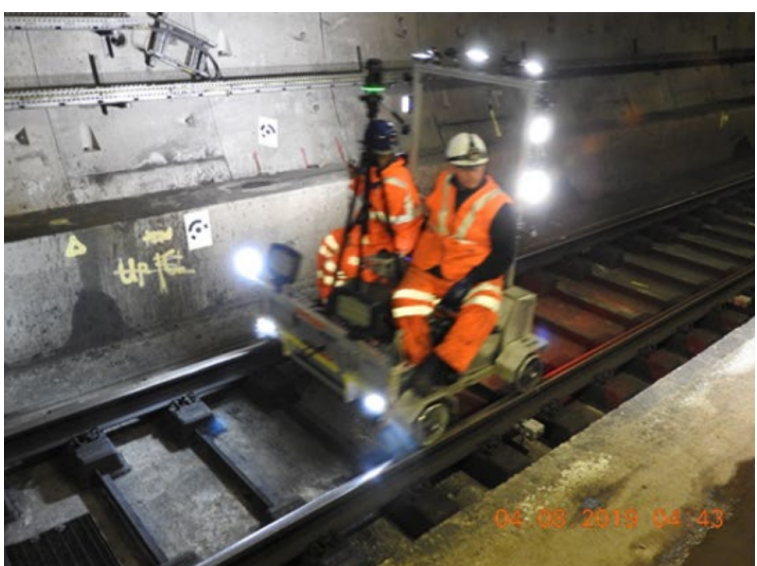

Figure 1. Test setup: camera mounted on the aluminium frame at the back of the vehicle together with the lighting system and laser scanner positioned between the two operators

as ground control points (GCPs). GCPs coordinates are measured from the aligned laser scans. Additional reference sensors, such as total station, are preferable for this task but that would severely limit the approach in terms of time (already restricted to the engineering hours - from 00:00 to 05:00), number of staff required for safety purposes. (London Underground, 2017), (Network Rail, n.d.),(Great Britain, Health and Safety Executive, 2016). To maximize the speed of the survey, all the sensors are installed on a battery powered rail vehicle (AlumiKart) as shown in Figure 1.

\subsection{Close range photogrammetry}

The imaging sensor is selected considering the smallest defect to be detected coherently with the infrastructure's accepted conditions. Crack width is the selected parameter to define tunnel's conditions because of the proven relationship between cracking and durability (Comite Euro-International du Beton, 1990), (The European Union Per Regulation, 2004), (Song et al., 2009), (Marchand, Samson, 2009), (Sun, 2011). For tunnels and underground structures, the limitation about crack width is not uniquely defined but varies depending on several aspect among which the geology, the chemical aggressivity of the material at the interface with the primary tunnel lining, the strength of concrete, the cover, the material, spacing, size and position of reinforcement embedded in the concrete, frequency of the induced stress (BTS, ICE, 2004) as well as on the owners' requirements. Given such complexity, for the purpose of this research the authors decide to fix the lower limit of crack width to $5 \mathrm{~mm}$ (or $3 \mathrm{~mm}$ in case of multiple cracks) according to "ease of repair" classification of damages (Burland, 1995) approach still in use in the construction industry for asset management. Together with other cameras, the GoPros lenses' resolving power is tested in the lab using 1951 Usaf resolution test chart (USAF, 1951 ) and proven to be capable of capturing $1.6 \mathrm{~mm}$ thick lines with a contrast transfer function (CTF - (Luhmann et al., 2013)) of 0.3 in the best lighting condition ( 0.22 averaging the results from different lighting conditions). However, according to the Nyquist sampling theory (Nyquist-Shannon Sampling Theorem, 2020) the minimum sampling theory equals two times the detected spatial frequency (eq 1):

$$
\begin{gathered}
\frac{1}{\frac{R P}{2}}=f=1.6 \mathrm{~mm} \\
f_{\text {Nyquist }}=f * 2=3.2 \mathrm{~mm}
\end{gathered}
$$

Where $f$ represents the minimum spatial frequency detected from the lab test $(\mathrm{RP}=0.3 \mathrm{lp} / \mathrm{mm}$ is the resolving power from resolution tests) and $f_{\text {Nyquist }}$ is the sampling frequency. Considering the sensitivity to light of small CCD sensors like the one installed in the GoPros, a lighting system to maximize the camera resolution is empirically designed and realized with 6 LED lights (5k lumen each) radially organised on the aluminium frame together with the GoPros (see Figure 1).

Two datasets are captured. The first one consists of still images (stop\&go)@approx $0.75 \mathrm{~m}$ (acquisition time $\approx 3 \mathrm{~min}$ ). The second dataset (video_mode) consists of videos simultaneously recorded at a speed of $1.4 \mathrm{~m} / \mathrm{s}$ (acquisition time $\approx 1.5 \mathrm{~min}$ for $100 \mathrm{~m}$ ); the speed is the lowest achievable with the rail vehicle used for the inspection. The resulting motion blur is calculated according to eq. 2 (Luhmann et al., 2013).

$$
\begin{aligned}
& \Delta s^{\prime}=\frac{\Delta t \cdot v}{m}=0.03 \mathrm{~mm} \\
& \Delta S=\Delta s^{\prime} \cdot m=46.30 \mathrm{~mm}
\end{aligned}
$$

where $\Delta s^{\prime}$ is the image blur in the image space, $\Delta t$ is the exposure time $(=1 / 30 \mathrm{sec}), v$ is the vehicle speed, $m$ is the image scale factor (average $\mathrm{m} \approx 1500$ ) and $\Delta S$ is the blur in the object space.

Two frames per second are extracted from each video in order to obtain a sufficient along-track overlap between consecutive frames. A quality comparison (considering image sharpness as quality parameter) between still imagery and frames extracted from videos is performed afterwords. There are several articles about this topic. For example, (Pertuz et al., 2013) reviews more than 30 methods to measure the focus of images. One of the possibilities could be to use the Fourier Transform (Fast Fourier Transform, 2020) evaluating image blur as function of the quantity of high frequencies. This approach could be challenging in a heterogeneous environment like tunnels. The present paper uses the Variance of Laplacian (Pech-Pacheco et al., 2000) to estimate the image blur. Laplacian operator (used to calculate the second derivate of the image) is often used for edge detection being able to identify rapid intensity changes. A high variance of Laplacian is indicative of an in focus image. On the contrary, a low variance of Laplacian denotes a lower ability to transfer edges and so the image is considered blur. In Figure 2 it is reported the variance of Laplacian averaged over the camera network for the two datasets. As expected, the stop\&go images show a consistently higher sharpness compared with video_mode ones. This, together with the motion blur estimation reported above, pushes the authors in choosing the still imagery photogrammetry for the purpose of the paper. However, ongoing research aims to demonstrate the applicability of videogrammetry for tunnels asset management.

Self camera calibration parameters is performed separately per each camera applying the Brown's model with commercial software AgisoftAgisoft Metashape.

The resulting point cloud shows a reprojection error distribution smaller than 4.4pixels (@2 $\sigma$ ) with a mean of 1.25pixels and a standard deviation of 1.73 pixels. Noteworthy, the biggest errors are focused in the zone surrounding the portal (see Figure 3) due to the presence of the corridor (perpendicular to the track so mainly captured in the images' peripheral area and with small reprojection angles) as well as the presence of passing operators in the images. 


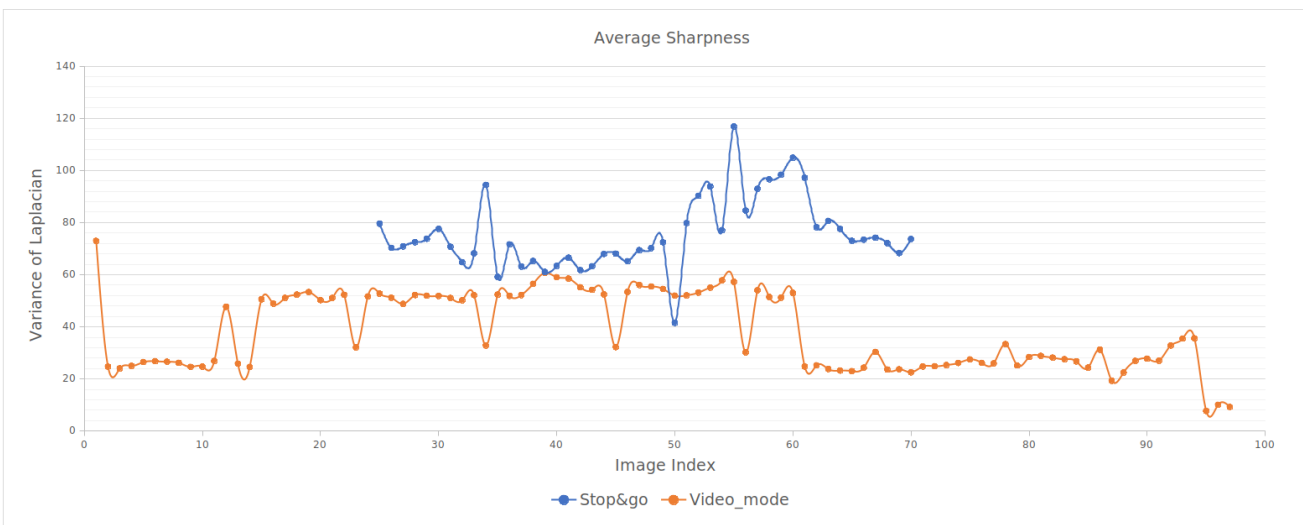

Figure 2. Image sharpness comparison between stop\&go dataset and video_mode dataset using Variance of Laplacian

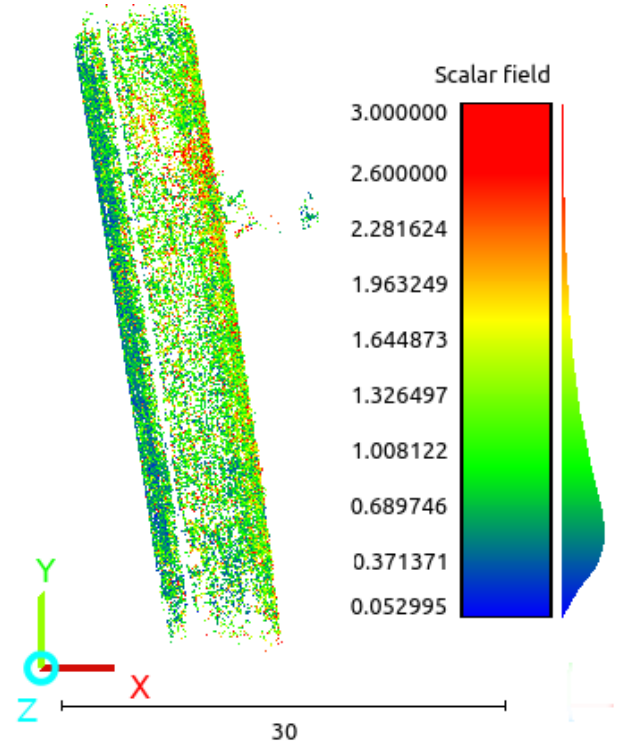

Figure 3. Reprojection error per point averaged over all images

Finally, the calculations proposed before about the resolving power of the lenses are confirmed by the point spacing of $2.2 \mathrm{~mm} /$ pixels calculated over a circular surface $(\mathrm{r}=1 \mathrm{~m})$ according to the following equation:

$$
\left\{\begin{array}{l}
\text { Density }=\frac{N_{\text {points }}}{\text { Area }} \\
\text { Point }_{\text {spacing }}=\sqrt{\frac{1}{\text { Density }}}
\end{array}\right.
$$

\subsection{TLS model}

The Leica BLK360 adopted for the TLS methodology performs $360^{\circ} 3 \mathrm{D}$ measurements with time of flight technology. Its nominal ranging accuracy is 4mm @ 10m / 7mm @20m (related 3D point cloud accuracy - at 78\% albedo=6mm @10m/8mm @ $20 \mathrm{~m})$. The information about the accuracy is used to design the maximum along-track distance between the targets (used to create a common reference system - see 3.1) and the distance between two consecutive scans (both set to 10m). This maximizes TLS target's 3D measurements and the overall 3D point accuracy. A total of 5 scans are recorded and aligned with the commercial software Leica Cyclone REGISTER 360. Given the repeatability of features for the inspected infrastructure, the automatic inbuilt alignment of the scans was not applicable but a manual pre alignment followed by a Rigid Iterative Closest Point (RICP) alignment optimization algorithm are performed. The model strength and the expected bundle error between the scans are confirmed by the software's outputs (Overlap 68\%, Strength $74 \%$, Bundle Error $5 \mathrm{~mm}$ ). The point spacing, calculated as stated in Equation 3, results $1.5 \mathrm{~mm}$.

\subsection{Comparisons}

3.4.1 Geometric accuracy The comparison between TLS and CRP techniques is performed at two levels. The first one detects target coordinates from the aligned TLS and uses them as GCPs for the 3D photogrammetric reconstruction. This might be questionable and additional reference sensors could be used instead. The reasons supporting such approach are reported in 3.1. The resulting root mean square error on the control points is $8 \mathrm{~mm}$. This value includes also the error coming from TLS registration $( \pm 5 \mathrm{~mm})$. The second level of the comparison is a cloud-to-cloud (C2C) distance assuming the TLS model as reference. $\mathrm{C} 2 \mathrm{C}$ distance is evaluated computing nearest neighbour distance according to Hausdorff distance algorithm (Gao, Dai, 2015) between the sparse photogrammetric point cloud and the laser scanner point cloud. Given the TLS model's high density the approximation of using nearest neighbour distance rather than the actual nearest point on the surface described by the TLS point cloud (see Figure 4) is considered negligible. Fig-

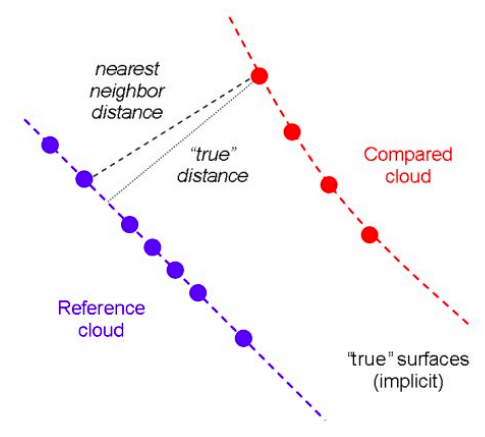

Figure 4. Nearest neighbour distance evaluation. Source

(Distances Computation - CloudCompareWiki, n.d.)

ure 5 together with Table 1 describe the comparison in terms of $\mathrm{C} 2 \mathrm{C}$ distances (directions according to the coordinate system reported in Figure 3$)$. Noticeable, at $\sigma(\approx 66 \%$ of data samples) the global distance is within the range of the scanner's accuracy. 


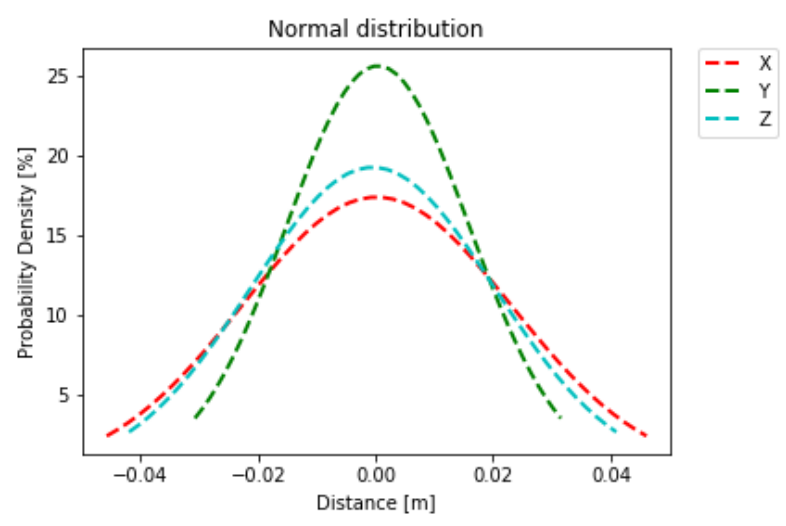

Figure 5. Normal distribution of the $\mathrm{C} 2 \mathrm{C}$ distances in the 3 directions.

\begin{tabular}{cccccc}
\hline Direction & mean & std & $\mathbf{5 0 \%}$ & $\mathbf{6 6 \%}$ & $\mathbf{9 5 \%}$ \\
\hline X & 0.0002 & 0.0229 & 0.0001 & 0.0013 & 0.0108 \\
Y & 0.0004 & 0.0156 & 0.0001 & 0.0006 & 0.0058 \\
Z & -0.0005 & 0.0207 & 0.0000 & 0.0004 & 0.0079 \\
ABS & 0.0105 & 0.0330 & 0.0031 & 0.0047 & 0.0398 \\
\hline
\end{tabular}

Table 1. C2C distances

3.4.2 Cost-Benefit In this section a cost-benefit analysis is reported. We decided to compare the proposed methodology with the routine visual inspection and routine detailed inspection (McKibbins et al., 2009).

In terms of costs, hardware represent usually less than $10 \%$ of the total budget allocated for visual inspections (this percentage decreases even more considering its reusability to a multitude of inspections) and for this reason it was not reported in this research paper. Instead, time, especially time spent on site for data acquisition or for manual data processing, has heavier weight in the computation of costs. The proposed methodology's bottleneck is represented by target placing. To speed up this step and to be able to easily remove any material/tool from the tunnel at the end of the shift, the markers were printed on adhesive paper and simply glued to the concrete lining. The application of targets to the lining was performed in 30 minutes (60 markers were applied to the lining since the original plan was to inspect $100 \mathrm{~m}$ of the tunnel). However, such delay is not considered in the comparison because markers are needed for both alignment purposes (cameras and laser scans - see 3.2 and 3.3) and to create a common reference system.

In Table 3 the acquisition and manual data processing times for the proposed methodologies are reported and compared against the traditional inspection approaches. The software computation time (23' 49" plus $3 \mathrm{~h} 45$ ' 52 " for matching\&alignment and dense point cloud generation respectively) is not considered because not influential on the costs.

A straight cost comparison was not possible between the traditional approaches and the proposed methods, so the total number of staff required per inspected ring per shift (4h max - see 3) was calculated (Table 3).

About the benefits, in 3.4.1 we already reported the geometric comparison between CRP and TLS. Moreover, CRP provides the user with photo-realistic outputs (see figure 6), easier to implement within the reports still mainly 2D. Furthermore, with the photogrammetric approach is easier to detect small defects from the high resolution images and project them into the $3 \mathrm{D}$ model. Doing so, it is possible to easily geo-referenciate in

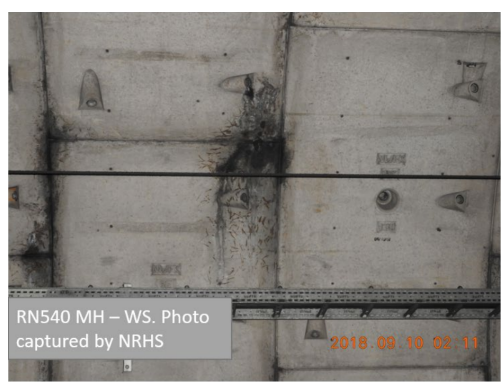

(a) Image captured by NRHS staff

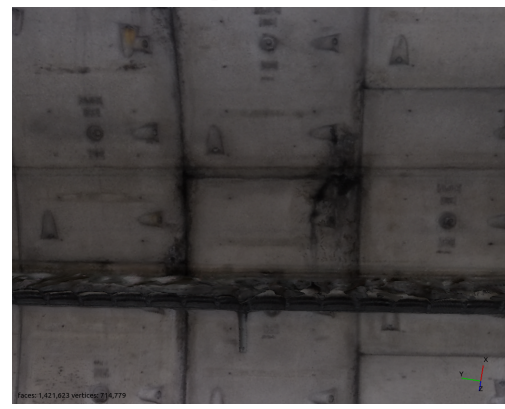

(b) Screenshot from the 3D model

Figure 6. Water ingress detection from (a) routine visual inspection and (b) 3D photogrammetric model.

local/global coordinates the detected defects of interest.

Finally, as a result of the collaboration with NRHS engineers, a visual interface is realised to interactively navigate the infrastructure thanks to a smooth transition between 2D and 3D. The proposed dashboard includes the 3D model, the 2D images and all the data of interest (see Figure 7) all in the same reference system (hence another reason to justify the presence of GCPs). The benefits reported so far, apart from the geometric comparison, are not measurable. For this reason, a qualitative comparison between the several inspection approaches is reported in Table 2. The values per each section range between 1 (nonfunctional) to 5 (fully functional). The final benefit score is a sum of all the addends. To the authors' opinion, the gain in benefits is too remarkable to be neglected and supplementing the inspection of tunnels with innovative technologies not only is possible but should be pursued.

\subsection{Conclusions and future research}

The research project described in this paper points out the potential of photogrammetry for tunnel inspections stressing to the limit the concept of low-end sensor adopting action cameras for the purpose of 3D photogrammetric reconstruction. This decision is explained and justified in 2. It demonstrates how a photogrammetric setup of $£ 3.5 \mathrm{k}$ can achieve comparable result with a £16k laser scanner (Leica BLK360 Imaging Laser Scanner, n.d.) in one fifth of the time (in terms of data acquisition cfr Table 3) with great direct effects on the costs and on H\&S conditions. In Figure 8 it is reported the normalized BenefitCost-Ratio (BCR) where the benefits are from Table 2 while the costs equal the inverse of number of rings per staff member (see Table 3). 


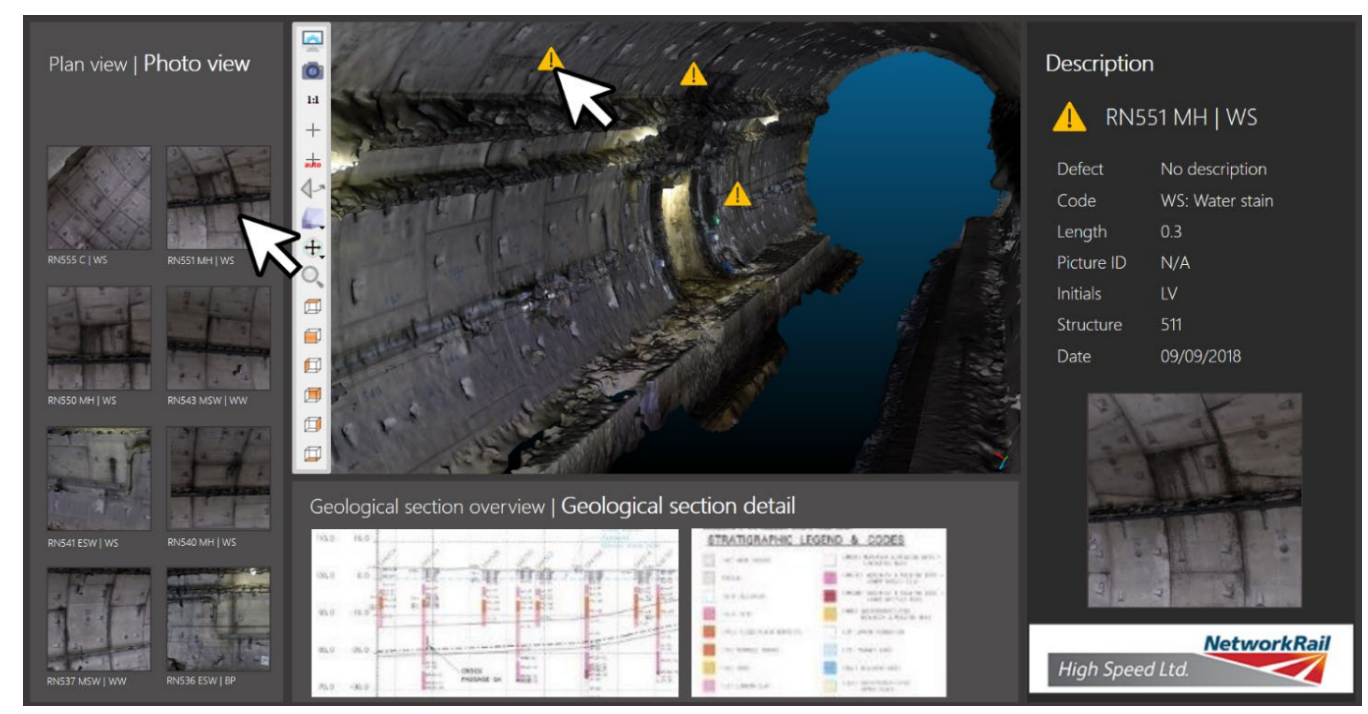

Figure 7. Dashboard developed from Arup for visual inspection of tunnels with photogrammetry

\begin{tabular}{lcccccc}
\hline Inspection Method & Geometry & repeatability & Objectivity & Data completeness & Automation & Benefit \\
\hline Visual & 1 & 2 & 2 & 3 & 1 & 9 \\
Detailed & 2 & 3 & 3 & 3 & 1 & 12 \\
CRP & 4 & 4 & 5 & 4 & 4 & 21 \\
TLS & 5 & 4 & 5 & 4 & 4 & 22 \\
\hline
\end{tabular}

Table 2. Qualitative comparison between the inspection methods (1: non-functional - 5: fully functional)

\begin{tabular}{lrrr}
\hline Method & \multicolumn{2}{c}{ Acquisition } & Processing* \\
& $@ 50 \mathrm{~m}$ & No. rings/staff & @ 50m \\
\hline Visual & - & 107 & - \\
Detailed & - & 31 & - \\
TLS & $25 \mathrm{~min}$ & 72 & 2 days \\
stop\&go & $3 \mathrm{~min}$ & 343 & $\frac{1}{2}$ day \\
\hline
\end{tabular}

Table 3. Time comparisons per each methodology

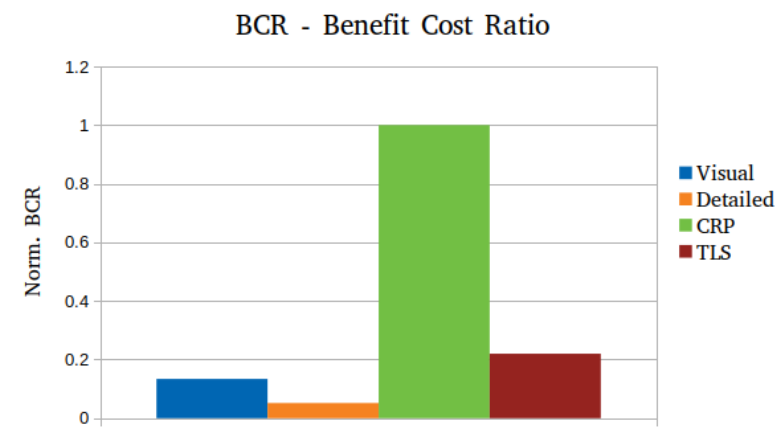

Figure 8. BCR for the different approaches

In the evaluation of costs it is not included the time to position the targets for several reasons. First of all, being GCPs needed in any inspection approach, it is auspicable that in the future markers will be embedded in the concrete lining likewise the prisms used for total station measurements. Moreover, the authors are already considering new strategies to go targetless with machine learning approaches to detect and use tunnels' features and their known design location (i.e. grout holes) as GCPs. This, combined with a precise block camera calibration could significantly increase BCR values.
The availability of Mobile Laser Scanners (MLS) technology is not new to the authors. However, it shall be pointed that in tunnels there is no GPS signal needed to map the data and a low end IMU (Inertial Measurement Unit) would prone to produce large drifts if external controls are not in place. There are multiple solutions like adopting a dynamic tracking system but this goes beyond the topic of the present research paper.

In conclusion, the authors argue that this results will push tunnel inspection in the direction of automated approaches with direct benefits on surveying costs as well as $\mathrm{H} \& \mathrm{~S}$. Utilising available technology supports risk-based asset management and thus ensures safe and operational performance of a railway for passengers to use.

\section{ACKNOWLEDGEMENTS}

The work carried out in this paper was developed thanks to the collaboration between Arup Ltd., Network Rail High Speed Ltd. and HS1 Ltd. The authors gratefully acknowledge mutual support. 


\section{REFERENCES}

Agisoft Metashape User Manual - Professional Edition, Version $1.5,2019$.

Balletti, C., Guerra, F., Tsioukas, V., Vernier, P., 2014a. Calibration of Action Cameras for Photogrammetric Purposes. Sensors (Basel, Switzerland), 14(9), 17471-17490.

Balletti, C., Vernier, P., Guerra, F., Tsioukas, V., 2014b. Calibration of Action Cameras for Photogrammetric Purposes. Sensors, 14(9).

Barla, G., Antolini, F., Gigli, G., 2016. 3D Laser scanner and thermography for tunnel discontinuity mapping. Geomechanics and Tunnelling, 9, 29-36.

Bauer, A., Gutjahr, K., Paar, G., Kontrus, H., Glatzl, R., 2015. Tunnel Surface 3D Reconstruction from Unoriented Image Sequences.

Brian, S., 1997. The Consequences of Poor Serviceability and the Way Forward. Management Of Concrete Structures For Long-Term Serviceability, Thomas Telford Publishing, 1-8.

Brown, D. C., 1971. Close-Range Camera Calibration. Photogrammetric Engineering, 37(8), 855-866.

BTS, T. B. T. S., ICE, T. I. o. C. E., 2004. Design and specification for durability. Tunnel Lining Design Guide, Thomas Telford Publishing, 1 Heron Quay, London E14 4JD.

Burland, J. B., 1995. Assessment of Risk of Damage to Buildings Due to Tunnelling and Excavation. 1st International Conference on Earthquake Geotechnical Engineering, Tokyo.

Centre, O. M., 2003. Handbook of practical camera calibration methods and models Optical Metrology Centre CHAPTER 4 CAMERA CALIBRATION METHODS.

Charbonnier, P., Chavant, P., Foucher, P., Muzet, V., Prybyla, D., Perrin, T., Grussenmeyer, P., Guillemin, S., 2013. Accuracy Assessment of a Canal-Tunnel 3d Model by Comparing Photogrammetry and Laserscanning Recording Techniques. ISPRS International Archives of the Photogrammetry, Remote Sensing and Spatial Information Sciences, 5, 171-176.

Comite Euro-International du Beton, 1990. CEB FIP Model Code.

Distances Computation - CloudCompareWiki, n.d. https://www.cloudcompare.org/doc/wiki/index.php?title= Distances $\backslash$ Computation.

Fahmy, M., Eng, P., Asce, M., Moselhi, O., Asce, F., 2009. Detecting and Locating Leaks in Underground Water Mains Using Thermography. 2009 26th International Symposium on Automation and Robotics in Construction, ISARC 2009.

Fassi, F., Troisi, S., Baiocchi, V., Del Pizzo, S., Giannone, F., Barazzetti, L., Previtali, M., Roncoroni, F., 2018. Fisheye Photogrammetry to Survey Narrow Spaces in Architecture and a Hypogea Environment. Latest Developments in Reality-Based $3 D$ Surveying and Modelling, MDPI.

Fast Fourier Transform, 2020. Fast Fourier Transform. Wikipedia. Page Version ID: 944528739.
Fort-González, R., López, H., Mingarro, F., 2002. Assessment of protective treatments based on their chromatic evolution: Limestone and granite in the Royal Palace of Madrid. Protection and Conservation of the Cultural Heritage of the Mediterranean Cities, 347-442.

Gao, Y., Dai, Q., 2015. Chapter 7 - learning-based 3-D object retrieval. Y. Gao, Q. Dai (eds), View-Based 3-d Object Retrieval, Morgan Kaufmann, Boston, 111-136.

González-Aguilera, D., Gómez-Lahoz, J., Sánchez, J., 2008. A New Approach for Structural Monitoring of Large Dams with a Three-Dimensional Laser Scanner. Sensors (Basel, Switzerland), 8(9), 5866-5883.

Great Britain, Health and Safety Executive, 2016. Manual Handling: Manual Handling Operations Regulations 1992, Guidance on Regulations L23. HSE Books, Place of publication not identified. OCLC: 959648937.

Gulvanessian, H., 2009. Eurocode 0: BS EN 1990: 2002. Basis of Structural Design. Thomas Telford, London. OCLC: 953540004

Herrera, L. K., Borgne, S. L., Videla, H. A., 2008. Modern Methods for Materials Characterization and Surface Analysis to Study the Effects of Biodeterioration and Weathering on Buildings of Cultural Heritage. International Journal of Architectural Heritage, 3(1), 74-91. _eprint: https://doi.org/10.1080/15583050802149995.

Kannala, J., Br, S., 2004. A generic camera calibration method for fish-eye lenses. International Conference on Pattern Recognition.

Leica BLK360 Imaging Laser Scanner, n.d. https://www.sccssurvey.co.uk/leica-blk360-imaging-laserscanner.html.

Lemy, F., Yong, S., Schulz, T., 2006. A Case Study of Monitoring Tunnel Wall Displacement Using Laser Scanning Techno$\operatorname{logy}$.

London Underground, L., 2017. Lu-ici-v1-january2017. http://content.tfl.gov.uk/lu-ici-v1-january-2017.pdf.

Luhmann, T., Robson, S., Kyle, S., Boehm, J., 2013. CloseRange Photogrammetry and $3 D$ Imaging. 2nd ed. edn, De Gruyter, Berlin, Boston.

M, T., D, L., N, P., n.d. Terrestrial Laser Scanning for Deformation Monitoring.

Maldonado, G., Newsome, S., Maghiar, M., Clendenen, J. T., Jackson, N. M., 2016. Discrepancy Analysis between CloseRange Photogrammetry and Terrestrial LiDAR.

Mandelli, A., Fassi, F., Perfetti, L., Polari, C., 2017. TESTING DIFFERENT SURVEY TECHNIQUES TO MODEL ARCHITECTONICNARROW SPACES. ISPRS - International Archives of the Photogrammetry, Remote Sensing and Spatial Information Sciences, XLII-2/W5, 505-511.

Marchand, J., Samson, E., 2009. Predicting the Service-Life of Concrete Structures - Limitations of Simplified Models. Cement and Concrete Composites, 31(8), 515-521. 
McDonnell, D., Devriendt, M., 2017. The Application of BIM and Photogrammetric Methods to the Inspection and Management of the Sydney Harbour Tunnel. 16th Australasian Tunnelling Conference 2017: Challenging Underground Space : Bigger, Better, More, 335.

McKibbins, L., Elmer, R., Roberts, K., 2009. Tunnels: Inspection, Assessment and Maintenace. CIRIA C, CIRIA, London. OCLC: 755982542.

Moisan, E., Heinkele, C., Charbonnier, P., Foucher, P., Grussenmeyer, P., Guillemin, S., Koehl, M., 2017. DYNAMIC 3D MODELING OF A CANAL-TUNNEL USING PHOTOGRAMMETRIC AND BATHYMETRIC DATA. ISPRS - International Archives of the Photogrammetry, Remote Sensing and Spatial Information Sciences, XLII-2/W3, 495-501.

Monserrat, O., Crosetto, M., 2008. Deformation Measurement Using Terrestrial Laser Scanning Data and Least Squares 3D Surface Matching. ISPRS Journal of Photogrammetry and Remote Sensing, 63(1), 142-154.

Network Rail, N., n.d. PTS-Personal-TrackSafety.pdf. http://www.fastlinetraining.co.uk/wpcontent/uploads/2016/11/PTS-Personal-Track-Safety.pdf.

Nyquist-Shannon Sampling Theorem, 2020. Nyquist-Shannon Sampling Theorem. Wikipedia. Page Version ID: 944809399.

Paar, G., Kontrus, H., 2007. Three-Dimensional Tunnel Reconstruction Using Photogrammetry and Laser Scanning.

Pech-Pacheco, J., Cristobal, G., Chamorro-Martinez, J., Fernandez-Valdivia, J., 2000. Diatom autofocusing in brightfield microscopy: A comparative study. Proceedings 15th International Conference on Pattern Recognition. ICPR-2000, 3, 314-317 vol.3.

Perfetti, L., Polari, C., Fassi, F., 2018. FISHEYE MULTICAMERA SYSTEM CALIBRATION FOR SURVEYING NARROW AND COMPLEX ARCHITECTURES. ISPRS - International Archives of the Photogrammetry, Remote Sensing and Spatial Information Sciences, XLII-2, 877-883.

Pertuz, S., Puig, D., García, M. Á., 2013. Analysis of Focus Measure Operators for Shape-from-Focus. Pattern Recognit.

Qiu, D., Gao, L., 2010. Application of virtual reality technology in bridge structure safety monitoring. 2010 International Conference on Computer and Information Application, 465-467.

Reznicek, J., Luhmann, T., 2019. Finite-Element Approach to Camera Modelling and Calibration. PFG - Journal of Photogrammetry, Remote Sensing and Geoinformation Science, 87(1), 1-17.

Shortis, M., 2019. Camera calibration techniques for accurate measurement underwater. J. K. McCarthy, J. Benjamin, T. Winton, W. van Duivenvoorde (eds), 3D Recording and Interpretation for Maritime Archaeology, Springer International Publishing, Cham, 11-27.

Song, H.-W., Shim, H.-B., Petcherdchoo, A., Park, S.-K., 2009. Service Life Prediction of Repaired Concrete Structures under Chloride Environment Using Finite Difference Method. Cement and Concrete Composites, 31(2), 120-127.

Sun, J., 2011. Durability Problems of Lining Structures for Xiamen Xiang'an Subsea Tunnel in China. Journal of Rock Mechanics and Geotechnical Engineering, 3(4), 289-301.
Teo, T., 2015. Video-Based Point Cloud Generation Using Multiple Action Cameras. ISPRS - International Archives of the Photogrammetry, Remote Sensing and Spatial Information Sciences, 4, 55-60.

The European Union Per Regulation, 2004. EN 1992-1-1.

USAF, 1951. 1951 USAF resolution test chart - Wikipedia. https://en.wikipedia.org/wiki/1951_USAF_resolution_test_chart.

Wierzbicki, D., 2018. Calibration Low-Cost Cameras with Wide-Angle Lenses for Measurements. Journal of Automation Mobile Robotics and Intelligent Systems, Vol. 12, No. 2.

Yu, P., Wu, H., Liu, C., Xu, Z., 2018. WATER LEAKAGE DIAGNOSIS IN METRO TUNNELS BY INTERGRATION OF LASER POINT CLOUD AND INFRARED THERMAL IMAGING. ISPRS - International Archives of the Photogrammetry, Remote Sensing and Spatial Information Sciences, XLII3, 2167-2171.

\section{APPENDIX}

Any additional supporting data may be appended, provided the paper does not exceed the limits given above.

Revised 29th May 2020 